\title{
Determinación de la actividad tóxica de extractos optimizados de Palmaria y Porphyra para el tratamiento de la lesión por isquemia/reperfusión
}

\author{
Determination of the toxic activity of optimized extracts of Palmaria and Porphyra \\ as a treatment for ischemia-reperfusion injury
}

Fecha de recepción y aceptación: 21 de febrero de 2020, 22 de mayo de 2020

DOI: $10.46583 /$ nereis_2020.12.579

\section{Polanco Irisarri1 ${ }^{*}$ y C. Padrón Sanz ${ }^{1}$}

${ }^{1}$ Facultad de Veterinaria y Ciencias Experimentales, Universidad Católica de Valencia San Vicente Mártir.

* Correspondencia: Universidad Católica de Valencia San Vicente Mártir. Facultad de Veterinaria y Ciencias Experimentales.

Calle Guillem de Castro 94.46001 Valencia. España.E-mail: david.polanco@mail.ucv.es

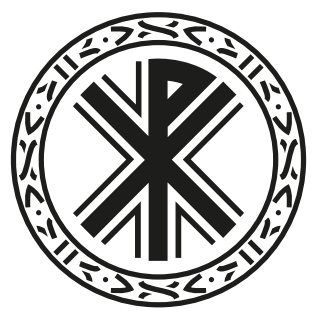

\section{RESUMEN}

La lesión por isquemia-reperfusión (I/R) puede provocar rechazos en los trasplantes de órganos debido al daño a nivel celular que producen los radicales libres de oxígeno (ROS). Se ha comprobado que los antioxidantes naturales pueden reducir este daño. Los extractos de muchas algas poseen una actividad antioxidante notable, pero se necesita comprobar la toxicidad de estos antes de proponerlos como fármacos. En este estudio, se realizaron dos ensayos toxicológicos con queratinocitos humanos inmortalizados (HaCaT) y nauplios de Artemia salina para valorar la toxicidad de dos extractos con actividad antioxidante de Palmaria palmata y Porphyra sp. Se obtuvieron $\mathrm{LC}_{50}$ de 7,843\% $(\mathrm{v} / \mathrm{v})$ de concentración de extracto en $P$. palmata en el ensayo con $\mathrm{HaCaT}$ y $3,2583 \%(v / v)$ en el ensayo con $A$. salina, mientras que con Porphyra $s p$. se obtuvo $10,7463 \%(v / v)$ en el ensayo con HaCaT y $6,5688 \%(v / v)$ en el ensayo con $A$. salina. Los niveles sin efecto adverso observable (NOAEL) obtenidos para cada alga en cada ensayo mostraron significativamente una mayor toxicidad del extracto de $P$. palmata que del de Porphyra $s p$. $(\mathrm{p}<0,05)$. Finalmente, se propuso un índice que tiene en cuenta la actividad antioxidante y la toxicidad de cada extracto para predecir su eficiencia in vivo, confirmando los resultados anteriores, y por ello se escogió el extracto de Porphyra sp. como el más idóneo para continuar con los ensayos preclínicos en el desarrollo de un fármaco para el tratamiento de la lesión por I/R.

PALABRAS CLAVE: antioxidantes, alga, HaCaT, MTT, artemia.

\section{ABSTRACT}

The ischemia/reperfusion (I/R) injury can cause rejections in organ transplantation surgeries due to the damage that radical oxigen species (ROS) produce in the cells. It has been proved that natural antioxidants can reduce this damage. Lots of algae extracts show a noticeable antioxidant activity, but there's a need to test their toxicity before suggesting them as potential drugs. In this study, two toxicologic assays using human immortalized keratinocytes (HaCaT) and Artemia 
salina nauplii were carried out to test the toxicity of two extracts that showed antioxidant activity from Palmaria palmata and Porphyra $s p . \mathrm{LC}_{50}$ values of $7.843 \%(v / v)$ of extract concentration with P. palmata in the HaCaT assay and $3.2583 \%(v / v)$ in the A. salina assay were obtained, while $10.7463 \%(v / v)$ of extract concentration for the HaCaT assay and $6.5688 \%(v / v)$ for the A. salina assay were obtained for Porphyra sp. The non-observed adverse effect level (NOAEL) obtained for each algae in each assay showed a significantly higher toxicity level in the $P$. palmata extract than in the Porphyra sp. extract $(\mathrm{p}<0.05)$. Lastly, an index which considers both antioxidant activity and toxicity of each extract was proposed for predicting their in vivo efficiency, supporting the previous results, so Porphyra sp. extract was considered the most suitable for going ahead with the preclinic assays towards the development of a drug for treating the I/R injury.

KEYWORDS: antioxidants, algae, HaCaT, MTT, artemia.

\section{INTRODUCCIÓN}

La lesión por isquemia/reperfusión (Ischemia/Reperfusion, en adelante $\mathrm{I} / \mathrm{R}$ ) ocurre cuando a un tejido animal se le priva de flujo sanguíneo -y por tanto de oxígeno- durante un periodo prolongado de tiempo y, a continuación, se restablece el flujo, lo que produce una gran cantidad de radicales libres de oxígeno (Radical Oxigen Species, en adelante ROS) en las células de dicho tejido [1].

La I/R es una de las principales complicaciones que pueden existir en una operación quirúrgica de trasplante de órgano [2]. Con anterioridad a la operación, el órgano extraído del donante sufre un proceso de hipoxia fría durante su conservación a $4^{\circ} \mathrm{C}$ hasta el momento de la operación [2]. Posteriormente, tanto el tejido vascular del paciente durante el proceso de clamping arterial como el del órgano que se va a trasplantar son propensos a sufrir $\mathrm{I} / \mathrm{R}$ en el momento de la intervención quirúrgica [4]. Finalmente, el paciente puede sufrir retardo en la función del injerto (Delayed Graft Function, DGF), consecuencia de haber sufrido daño celular durante el trasplante, y el órgano puede perder su función e incluso ser rechazado [5].

Con el fin de evitar la formación de ROS, o al menos paliar sus efectos a nivel celular, diversos estudios $[2,6,7]$ sugieren que los antioxidantes son capaces de inhibir en gran medida el daño oxidativo causado por la I/R. Los antioxidantes son sustancias capaces de aceptar los electrones desapareados de los ROS, evitando así que estos oxiden otras moléculas en el tejido [8].

Existen dos clases de compuestos antioxidantes atendiendo a su origen. Los antioxidantes endógenos o enzimáticos son sintetizados por el mecanismo de transcripción/traducción celular, como es el caso de los enzimas catalasa, peróxido dismutasa, glutatión peroxidasa, reductasas, etcétera [9]. La otra clase de antioxidantes son los llamados exógenos o no enzimáticos, que no tienen un origen peptídico [10].

Los antioxidantes exógenos o no enzimáticos pueden ser naturales o sintéticos, siendo los de origen natural, por lo general, menos dañinos para los organismos [11], y habiéndose reportado en algún caso una mayor capacidad antioxidante en comparación con los sintéticos [12,13]. Además, los antioxidantes sintéticos en ocasiones son capaces de causar alergias o carcinomas debido a su toxicidad [14]. 
Dentro de los antioxidantes exógenos naturales se pueden distinguir varias familias de sustancias: vitaminas, pigmentos, polifenoles y polisacáridos sulfatados [15]. Estas moléculas son producidas por los organismos vivos fotosintéticos como una primera defensa ante agresiones oxidativas externas [16].

Utilizando el extractante adecuado, estas sustancias pueden ser extraídas de diversos organismos fotosintéticos, tanto de plantas vasculares [12,17-21] como de algas [6,22-24], y se ha demostrado que el extracto resultante puede tener una actividad antioxidante que puede ser utilizada para paliar el daño por I/R.

En este sentido, en estudios de experimentación animal con ratas, se ha comprobado que los extractos metanólicos de galio (Galium verum) pueden evitar lesiones cardiovasculares derivadas de la I/R (18) y los de granado (Punica granatum) son capaces de proteger el tejido nervioso del cerebro de la formación de ROS [20]. Los extractos butanólicos de corteza de manglar también han mostrado potencial para el tratamiento de lesiones estomacales causadas por I/R [19].

Existen algunos trabajos en los que se pone de manifiesto la capacidad de los extractos o compuestos extraídos de algas para paliar la lesión por I/R. Por una parte, los fucoidanos del alga parda Fucus vesiculosus han mostrado su eficacia frente a la apoptosis y la autofagia de las células hepáticas de ratones cuando se ven sometidas a la lesión por I/R [22]. Del mismo modo, los extractos organoacuosos de Himanthalia elongata han mostrado un gran potencial en el tratamiento de la lesión intestinal por I/R en ratas [6]. Por otra parte, se ha visto que el bromofenol extraído de algas rojas posee una notable capacidad antioxidante frente a los radicales libres generados en la lesión por I/R en cardiomiocitos de embriones de rata [25]. Los polisacáridos presentes en Porphyra yezoensis, los porfiranos, también pueden paliar el daño por estrés oxidativo en el tejido nervioso de ratas [26]. Se demostró que un extracto de Palmaria palmata diluido al $1 \%$ en gel no presenta citotoxicidad ni fototoxicidad en una monocapa de fibroblastos [27]. Aun así, se desconoce en gran medida el potencial antioxidante que pueden llegar a tener los extractos brutos de algas rojas y su posterior aplicación como fármaco.

Asimismo, es imprescindible realizar además una serie de test toxicológicos, ya que, a pesar de que muchos extractos vegetales o algales con capacidad antioxidante tienen capacidad para prevenir o paliar los efectos de la lesión por $\mathrm{I} / \mathrm{R}$, es necesario someter al extracto a una serie de ensayos previos para comprobar que no es tóxico para el organismo que se está tratando [28,29].

En este sentido, la línea celular HaCaT (queratinocitos humanos inmortalizados) se ha utilizado ampliamente en ensayos de crecimiento y diferenciación celular [30], aunque se utilizan también para detectar actividad citotóxica y antitumoral en diversos compuestos y extractos. Concretamente, se ha utilizado tanto en ensayos de extractos de plantas [15,31,32] como de algas [33,34].

Se comprobó que las líneas celulares inmortalizadas procedentes de tumores pueden utilizarse para detectar citotoxicidad en extractos de cianobacterias [35]. También se ensayaron extractos de algas rojas utilizando líneas celulares inmortalizadas, y se averiguó que los procedentes de Asparagopsis armata, Brongniartella byssoides y Heterosiphonia plumosa mostraban actividad citotóxica [36].

Por otro lado, se llevaron a cabo ensayos toxicológicos con nauplios de Artemia salina en un trabajo en el que se midió la toxicidad de los extractos de 29 plantas vasculares colombianas, de las que se extrajeron por separado varias familias de compuestos de interés toxicológico (alcaloides, flavonoides, taninos, nafta y/o antraquinonas, saponinas, esteroides y/o triterpenoides, lactonas terpénicas, cumarinas y cardiotónicos) y se evaluó el carácter tóxico de cada fracción por separado [37]. 
También se utilizaron nauplios, en este caso de 48 horas de vida, para medir la toxicidad de extractos etanólicos resuspendidos en DMSO de varias especies del género Solanum procedentes de Brasil [38].

Recientemente, también se han utilizado nauplios recién eclosionados para medir la toxicidad de extractos metanólicos de Bacopa monnieri [39] y de extractos acuosos de Moringa oleifera, una planta medicinal hindú [40].

En otro trabajo, se analizó la toxicidad de 38 extractos metanólicos y acuosos de macroalgas pardas, rojas y verdes de la costa coreana con el fin de detectar actividad tóxica para invertebrados [41].

Por todo lo expuesto anteriormente queda patente el interés en emplear ensayos de toxicidad aguda con queratinocitos de la línea celular HaCaT y con nauplios de Artemia salina en la detección de la posible actividad citotóxica de los extractos de algas.

Finalmente, el objetivo de este estudio es determinar la toxicidad de los extractos de Palmaria palmata y Porphyra sp. de manera previa a su empleo en el tratamiento de la lesión por isquemiareperfusión utilizando como indicadores de toxicidad de los extractos la concentración letal al $50 \%$ (Lethal Concentration at $50 \%, \mathrm{LC}_{50}$ ) y el nivel sin efecto adverso observable (No Observed Adverse Effect Level, NOAEL) de dichos extractos en la línea celular HaCaT (queratinocitos humanos inmortalizados) y en nauplios en estadio instar II de Artemia salina. Por otro lado, se pretende proponer un índice que tenga en cuenta tanto la actividad antioxidante como la actividad tóxica de los extractos obtenidos, calculada a partir de la NOAEL y de la $\mathrm{LC}_{50}$ obtenidas en los ensayos anteriores, y con el que se pueda comparar la eficiencia potencial de cada alga en cada ensayo realizado.

\section{MATERIAL Y MÉTODOS}

\section{Obtención de extractos y actividad antioxidante}

Los extractos de Palmaria palmata y Porphyra sp. se obtuvieron con un extractante organoacuoso aplicando en cada caso las condiciones óptimas para obtener la mayor actividad antioxidante (datos pendientes de publicación - tesis doctoral de Samanta García Oms, en proceso de realización).

La actividad antioxidante de los extractos obtenidos de las algas P. palmata y Porphyra sp. fue analizada mediante el test de DPPH [42] mostrando una actividad de 78,7 y 87,63 \% respectivamente.

A continuación, $5 \mathrm{~mL}$ de cada extracto fueron rotavaporados durante 20 minutos a $50{ }^{\circ} \mathrm{C}$ bajo presión negativa para retirar el extractante. El extracto seco fue resuspendido en $5 \mathrm{~mL}$ de DMEM para el ensayo MTT-24h, o en 5 mL de solución salina para el ensayo Artemia-24h.

\section{Línea celular}

Los queratinocitos humanos inmortalizados (HaCaT) se obtuvieron del Instituto de Investigación Príncipe Felipe (Valencia) y fueron cultivados en monocapa en flasks de cultivo celular con DMEM (High glucose, BioWest) en una incubadora de dióxido de carbono (Memmert, Tecnigen). El medio se cambió dos veces por semana. 


\section{Nauplios de Artemia salina}

Los nauplios de Artemia salina fueron obtenidos a partir de quistes comerciales (ARTEMIA-mix, SERA) y eclosionados en un medio salino [43,44] que contenía: $\mathrm{NaCl}, 23 \mathrm{~g} / \mathrm{L} ; \mathrm{MgCl} 2 \cdot 6 \mathrm{H} 2 \mathrm{O}$, $11 \mathrm{~g} / \mathrm{L} ; \mathrm{CaCl} 2 \cdot 6 \mathrm{H} 2 \mathrm{O}, 2 \mathrm{~g} / \mathrm{L} ; \mathrm{y} \mathrm{KCl}, 0,7 \mathrm{~g} / \mathrm{L}$, con el pH ajustado a 8,5 con $\mathrm{NaOH}$, e incubados a una temperatura de $26^{\circ} \mathrm{C}$.

\section{Ensayos de toxicidad MTT-24h con células humanas (HaCaT)}

La toxicidad de los extractos de Palmaria palmata y Porphyra sp. se monitorizó utilizando el ensayo MTT en queratinocitos humanos inmortalizados (HaCaT) siguiendo el protocolo de Li et al. [31]. Las células, cultivadas en monocapa en un flask, fueron puestas en suspensión tras un tratamiento con tripsina comercial $\left(10 \mathrm{x}, 1 \mathrm{~mL}, 10 \mathrm{~min}, 37^{\circ} \mathrm{C}\right.$ ) y diluidas en $4 \mathrm{~mL}$ de DMEM (High glucose, BioWest). Se homogeneizaron suavemente, se tomó una alícuota de $20 \mu \mathrm{L}$ a la que se añadieron $20 \mu \mathrm{L}$ de azul tripán, y se hizo un recuento con cámara Neubauer. Se tomó el volumen requerido de la suspensión madre de células para obtener una población de 10.000 células por pocillo de experimentación, se diluyeron dichas células en DMEM hasta obtener un volumen de $6 \mathrm{~mL}(100 \mu \mathrm{L}$ por pocillo de experimentación), se homogeneizó suavemente la dilución obtenida y se dispusieron $100 \mu \mathrm{L}$ por pocillo en los 60 pocillos centrales de placas de 96 pocillos para eliminar el efecto borde.

Transcurridas 24 h, se comprobó la adherencia de las células al fondo de los pocillos con un microscopio invertido de contraste de fases y se retiró el medio con una pipeta Pasteur de vidrio con cuidado de no dañar la monocapa. Se dispensaron $100 \mu \mathrm{L}$ con una concentración de 100, 50, 25, 10, 5 y $1 \%(v / v)$ del extracto diluido en DMEM en cada pocillo.

Tras un periodo de $24 \mathrm{~h}$, se retiró el medio con una pipeta Pasteur de vidrio y se dispensaron $100 \mu \mathrm{L}$ de una dilución de MTT (1:11 en DMEM). Tras incubar durante $3 \mathrm{~h}$, se retiró el MTT y se añadieron $100 \mu \mathrm{L}$ de dimetilsulfóxido (DMSO, $\mathrm{CH}_{3} \mathrm{SOCH}_{3}, \geq 99,9 \%$, Fischer Chemical) a cada pocillo para disolver los cristales formados. Se midió la absorbancia de los pocillos a $600 \mathrm{~nm}$ con un lector de placas (Varioskan Lux, Thermo Scientific).

Los resultados de viabilidad celular a partir de la actividad mitocondrial se expresaron en porcentaje $(\%)$ de absorbancia respecto al control, como indica la siguiente ecuación:

$$
\text { Mitochondrial Activity }(\%)=\left(\frac{A_{\text {TREATMENT }}}{A_{\text {CONTROL }}}\right) * 100
$$

donde $A_{\text {TREATMENT }}$ es la absorbancia de cada pocillo experimental y $A_{C O N T R O L}$ es la absorbancia media del control. Todos los procedimientos fueron llevados a cabo en una cabina de flujo laminar y bajo condiciones de esterilidad. 


\section{Ensayos de toxicidad Artemia-24h con nauplios de Artemia salina}

La toxicidad de los extractos de Palmaria palmata y Porphyra sp. se monitorizó utilizando el ensayo de toxicidad con Artemia salina [44]. Los nauplios fueron mantenidos en una solución salina modificada [43] que contenía: $\mathrm{NaCl}, 23 \mathrm{~g} / \mathrm{L} ; \mathrm{MgCl}_{2} \cdot 6 \mathrm{H}_{2} \mathrm{O}, 11 \mathrm{~g} / \mathrm{L} ; \mathrm{CaCl}_{2} \cdot 6 \mathrm{H}_{2} \mathrm{O}, 2 \mathrm{~g} / \mathrm{L} ; \mathrm{y} \mathrm{KCl}$, $0,7 \mathrm{~g} / \mathrm{L}$, con el pH ajustado a 8,5 utilizando $\mathrm{NaOH} 10 \%$, y a una temperatura de $26^{\circ} \mathrm{C}$.

Combinando los métodos de Sarabia [45] y de González y Gilling [46], se dispusieron $10 \pm 2$ nauplios en estadio instar II en placas de 48 pocillos que contenían $400 \mu \mathrm{L}$ del 100, 50, 25, 10, 5 y $1 \%$ $(v / v)$ del extracto diluido en solución salina en cada pocillo.

Tras 24 horas, se hizo un recuento de los nauplios que presentaban motilidad en cada pocillo. Los animales que no presentaban movimiento de los apéndices o del tracto digestivo se contabilizaron como muertos. En caso de que el número de nauplios en un pocillo fuese diferente a 10, se calculó proporcionalmente el porcentaje de mortalidad.

Los resultados de supervivencia de los nauplios se expresaron en porcentaje (\%) de supervivencia respecto al control, como indica la siguiente ecuación:

$$
\text { Survival }(\%)=\left(\frac{S_{\text {TREATMENT }}}{S_{\text {CONTROL }}}\right) * 100
$$

donde $S_{\text {TREATMENT }}$ es la supervivencia de cada pocillo experimental y $S_{\text {CONTROL }}$ es la supervivencia media del control $(>95 \%)$.

Como control positivo de toxicidad en los ensayos con A. salina, se realizó paralelamente un ensayo con dicromato potásico $\left(\mathrm{K}_{2} \mathrm{Cr}_{2} \mathrm{O}_{7},>99,9 \%\right.$, Merck) disuelto en solución salina a las concentraciones de 20, 15, 10 y 5 g/mL, según el método de González y Gilling (2001).

\section{Propuesta de Índice de Eficiencia Antioxidante (IEA)}

Con el fin de comparar el potencial farmacológico de los extractos de ambas algas, teniendo en cuenta tanto la capacidad antioxidante como la toxicidad que muestra cada uno, se propuso un Índice de Eficiencia Antioxidante que multiplica el porcentaje de capacidad antioxidante de cada extracto por cada uno de los dos parámetros toxicológicos, NOAEL y $\mathrm{LC}_{50}$, obtenidos previamente en cada tipo de ensayo.

El cálculo de los índices se realiza de la siguiente manera:

$$
\begin{gathered}
\mathrm{IEA}_{\mathrm{NOAEL}}=\mathrm{AA} \times \mathrm{NOAEL} \\
\mathrm{IEA}_{\mathrm{LC} 50}=\mathrm{AA} \times \mathrm{LC}_{50}
\end{gathered}
$$

donde IEA $_{\mathrm{NOAEL}}$ e IEA $_{\mathrm{LC50}}$ son, respectivamente, los índices de eficiencia antioxidante de la NOAEL y de la $\mathrm{LC}_{50}$ de cada extracto para cada ensayo y AA es la actividad antioxidante de cada extracto 
expresada en porcentaje. El resultado de ambas operaciones está expresado en porcentaje de actividad antioxidante por concentración $(\%, v / v)$ del extracto inicial.

Se compararon, dos a dos, el IEA de cada parámetro toxicológico, NOAEL y $\mathrm{LC}_{50}$, y cada ensayo. Puesto que unos valores altos de tanto la actividad antioxidante como los parámetros toxicológicos calculados aumentan el potencial farmacológico de los extractos, el extracto con el IEA más alto se consideró el más apto para continuar con los ensayos preclínicos.

Todos los ensayos se llevaron a cabo siguiendo el Reglamento (CE) n. ${ }^{\circ}$ 1907/2006 del Parlamento Europeo y del Consejo, de 18 de diciembre de 2006, relativo al registro, la evaluación, la autorización y la restricción de las sustancias y preparados químicos (UE, 2006), y el Reglamento (CE) n. ${ }^{\circ}$ 440/2008 de la Comisión de 30 de mayo de 2008 por el que se establecen métodos de ensayo de acuerdo con el Reglamento anterior (UE, 2008).

\section{Análisis estadístico}

Los datos recogidos fueron estadísticamente analizados utilizando un test de varianza de una vía (ANOVA) de SPSS (versión 17.0) con un p-valor $=0,05$ para hallar el nivel sin efecto adverso observable (NOAEL) para cada ensayo y extracto, y se representaron los valores medios \pm desviación estándar ( $\mathrm{n}=12$ en MTT-24h y n $=6$ en Artemia-24h).

Asimismo, se realizó, partiendo de los datos anteriores, un análisis de regresión probit para averiguar la $\mathrm{LC}_{50}$ de cada ensayo y extracto.

\section{RESULTADOS}

\section{Ensayo de toxicidad MTT-24h}

Los resultados del ensayo de toxicidad de los extractos de Palmaria palmata y Porphyra $s p$. en queratinocitos humanos inmortalizados se muestran en la figura 1. Como puede apreciarse, el NOAEL del extracto de P. palmata en queratinocitos humanos inmortalizados es menor que un $1 \%$ $(v / v)$ del extracto inicial, mientras que en el caso de Porphyra sp. es de un $1 \%$. Se puede descartar que el excipiente sea el factor causante de la toxicidad observada, ya que los grupos Control y Excipiente no presentan diferencias significativas $(\mathrm{p}<0,05)$.

Los resultados del análisis probit del ensayo MTT-24h para Palmaria palmata y Porphyra sp. se muestran en la figura 2 , y mostraron unas $\mathrm{LC}_{50}$ de $7,8743 \%$ y $10,7463 \%(v / v)$ del extracto, respectivamente. 

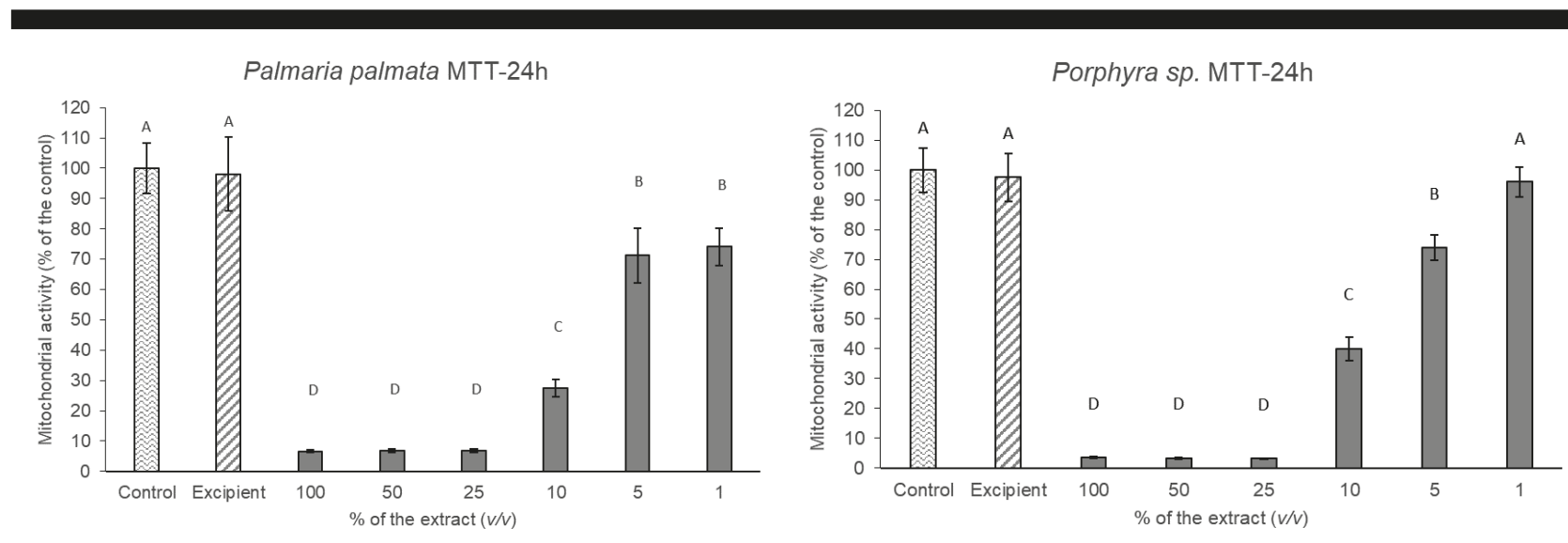

Fig. 1. Efecto de los extractos de Palmaria palmata y Porphyra sp. sobre la actividad mitocondrial de queratinocitos humanos inmortalizados (ensayo MTT). Las células fueron sembradas a razón de $10^{4}$ células/pocillo en placas de 96 pocillos, incubadas durante 24 horas $\left(5 \% \mathrm{CO} 2,100 \% \mathrm{RE}, 37^{\circ} \mathrm{C}\right)$ con dilución del extracto. El MTT reducido fue disuelto utilizando DMSO, y la absorbancia se midió a $600 \mathrm{~nm}$. Se muestran las medias de la actividad mitocondrial relativa al control \pm desviación estándar $(\mathrm{n}=12)$. Las letras mayúsculas indican diferencias significativas $(\mathrm{p}<0,05)$ en la actividad mitocondrial de cada concentración $(\%, v / v)$ del extracto.
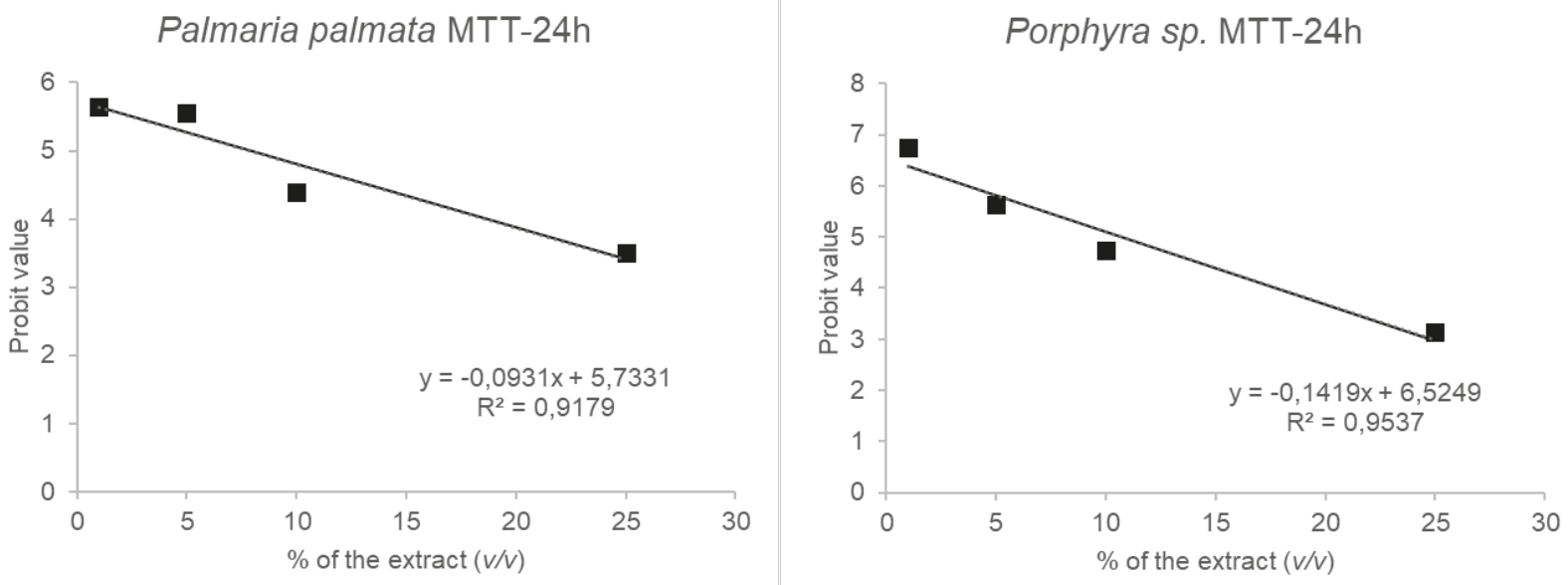

Fig. 2. Análisis probit del efecto del extracto de Palmaria palmata y Porphyra sp. sobre la actividad mitocondrial de queratinocitos humanos inmortalizados (ensayo MTT-24h). Los datos mostrados corresponden

a las concentraciones del $1,5,10$ y $25 \%(v / v)$ de extracto.

Estos resultados confirman lo observado en el análisis de los NOAEL, ya que la $\mathrm{LC}_{50}$ del extracto de $P$. palmata es inferior a la $\mathrm{LC}_{50}$ de Porphyra sp., lo que sugiere que el extracto de $P$. palmata presenta mayor toxicidad frente a queratinocitos humanos inmortalizados (HaCaT).

Los resultados del ensayo de toxicidad de los extractos de Palmaria palmata y Porphyra sp. en nauplios de Artemia salina se muestran en la figura 3. Como puede apreciarse, el NOAEL del extracto de $P$. palmata para nauplios de $A$. salina en estadio instar II es menor que una concentración del $1 \%$ 
$(v / v)$ de extracto, mientras que para Porphyra sp. es del $5 \%$. Se puede descartar que el excipiente sea el factor causante de la toxicidad observada, ya que los grupos Control y Excipiente no presentan diferencias significativas $(\mathrm{p}<0,05)$.
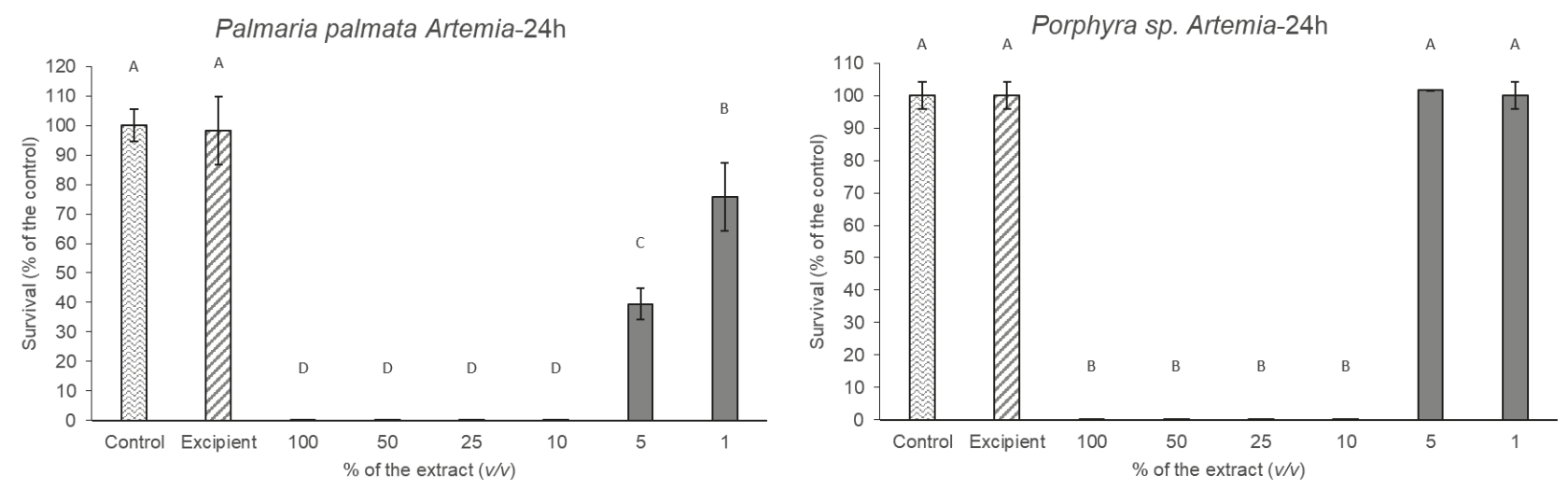

Fig. 3. Efecto de los extractos de Palmaria palmata y Porphyra sp. sobre la supervivencia de larvas (nauplios) de Artemia salina. Los nauplios fueron dispuestos a razón de $10 \pm 2$ nauplios/pocillo en placas de 48 pocillos, e incubados durante 24 horas $\left(100 \% \mathrm{RE}, 37^{\circ} \mathrm{C}\right)$ con dilución del extracto. Se observó el porcentaje de nauplios que presentaban motilidad como porcentaje de supervivencia en cada pocillo. Se muestran las medias de supervivencia de los nauplios relativa al control \pm desviación estándar $(\mathrm{n}=6)$. Las letras mayúsculas indican diferencias significativas $(\mathrm{p}<0,05)$ en la supervivencia de los nauplios a cada concentración $(v / v)$ del extracto.

Los resultados del análisis probit del ensayo Artemia-24h para Palmaria palmata y Porphyra sp. se muestran en la figura 4, y mostraron unas $\mathrm{LC}_{50}$ de 3,2583 y 6,5688 \% del extracto, respectivamente.

Palmaria palmata Artemia-24h

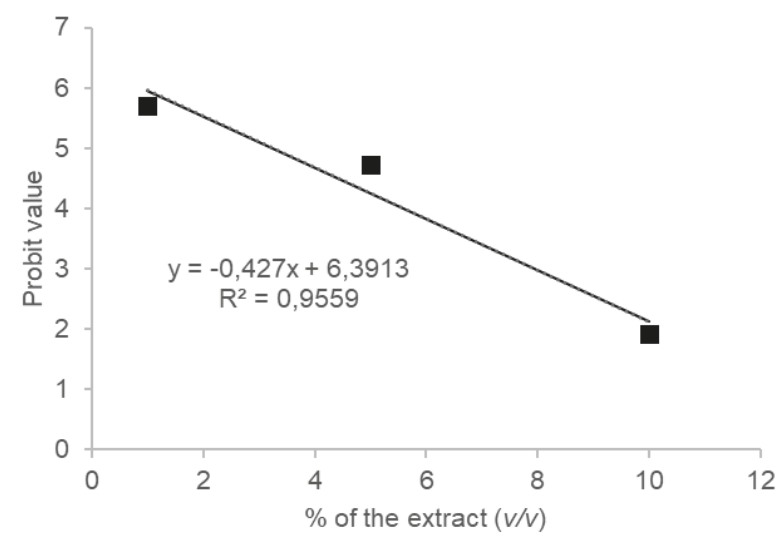

Porphyra sp. Artemia-24h

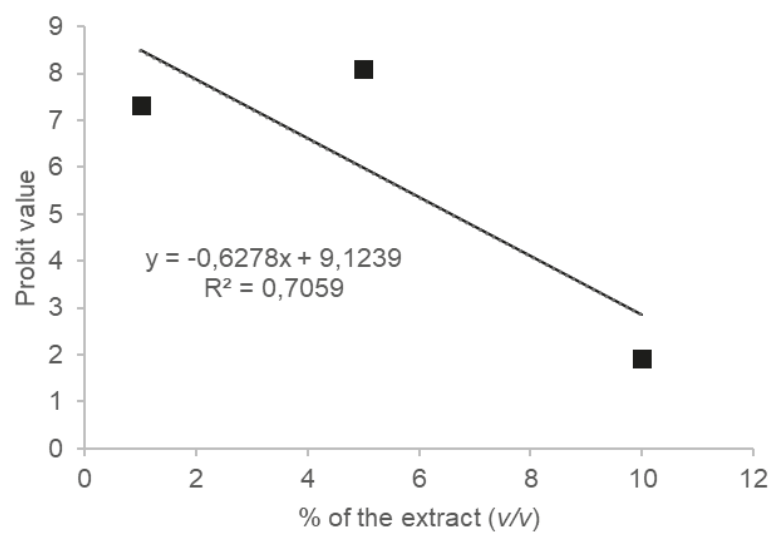

Fig. 4. Análisis probit del efecto del extracto de Palmaria palmata y Porphyra sp. sobre la supervivencia de nauplios de Artemia salina (ensayo Artemia-24h). Los datos mostrados corresponden a las concentraciones del 1, 5 y $10 \%$

$(v / v)$ de extracto. 
Estos resultados confirman lo observado en el análisis de los NOAEL, ya que la $\mathrm{LC}_{50}$ del extracto de P. palmata es inferior a la $\mathrm{LC}_{50}$ de Porphyra sp., lo que sugiere que el extracto de P. palmata presenta mayor toxicidad frente a nauplios de A. salina en estadio instar II, aunque sería necesario realizar un screening más fino entre los valores de 10 y $1 \%(v / v)$ de concentración de extracto que asegurase una distribución más ajustada a una función probit de los datos de mortalidad, sobre todo en el caso de Porphyra sp. $\left(\mathrm{R}^{2}=0,7059\right)$. Los resultados del control positivo con dicromato potásico mostraron una $\mathrm{LC}_{50}$ de $7,7304 \mu \mathrm{g} / \mathrm{mL}$.

Los resultados de NOAEL, $\mathrm{LC}_{50}$ e IEA calculados para cada alga en cada ensayo se muestran en la tabla 1.

Tabla 1. NOAEL, $\mathrm{LC}_{50}$ e IEA para cada alga en cada ensayo. Ambos índices están referidos a la actividad antioxidante que arroja cada extracto con el protocolo de extracción llevado a cabo. Los datos de NOAEL y LC $_{50}$ están expresados en $\%$ de concentración $(v / v)$ del extracto correspondiente

\begin{tabular}{ccccc}
\hline & \multicolumn{2}{c}{ MTT-24h } & \multicolumn{2}{c}{ Artemia-24h } \\
\cline { 2 - 5 } & P. palmata & Porphyra sp. & P. palmata & Porphyra sp. \\
\hline NOAEL $(\mathrm{p}<0,05)$ & $<1$ & 1 & $<1$ & 5 \\
$\mathrm{~L}_{\mathrm{CS}} 0$ & 7,8743 & 10,7463 & 3,2583 & 6,5688 \\
$\mathrm{IEA}_{\mathrm{NOAEL}}$ & $<78,7$ & 87,63 & $<78,7$ & 438,15 \\
$\mathrm{IEA}_{\mathrm{LC50}}$ & 619,70 & 941,69 & 256,42 & 575,62 \\
\hline
\end{tabular}

Como se ha descrito anteriormente, los niveles sin efecto adverso observable (NOAEL) obtenidos para el extracto de Palmaria palmata dan como resultado $<1 \%$ (MTT-24h) y $1 \%$ (Artemia-24h) de concentración $(v / v)$ de extracto, mientras que en el caso del extracto de Porphyra sp. dan como resultado $1 \%$ (MTT-24h) y $5 \%$ (Artemia-24h) de concentración $(v / v)$ de extracto que, comparando por separado los resultados de cada ensayo, permiten afirmar que hay indicios de que el extracto de P. palmata presenta más toxicidad que el extracto de Porphyra sp.

Por otra parte, las $\mathrm{LC}_{50}$ obtenidas para el extracto de P. palmata dan como resultado 7,8743\% (MTT-24h) y 3,2583\% (Artemia-24h) de concentración $(v / v)$ de extracto, mientras que en el caso del extracto de Porphyra sp. dan como resultado 10,7463\% (MTT-24h) y 6,5688 \% (Artemia-24h) de concentración $(v / v)$ de extracto que, comparando por separado los resultados de cada ensayo, corroboran los resultados obtenidos en el cálculo de los NOAEL.

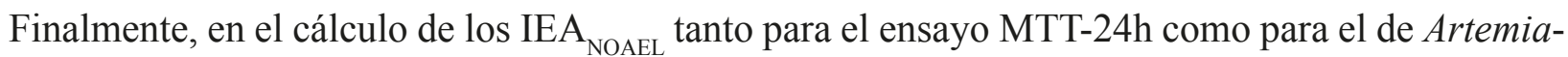
24h para cada ensayo se pueden observar valores más altos en el caso del extracto de Porphyra sp. que el de Palmaria palmata.

Como en el caso anterior, la comparación de los IEA $_{\text {LC50 }}$ tanto para el ensayo MTT-24h como para el de Artemia-24h para cada ensayo se obtuvo un valor más alto para el extracto de Porphyra sp. que para el de $P$. palmata, lo que permitiría confirmar que Porphyra sp. es el alga más adecuada para continuar con los ensayos en lo que respecta a la actividad antioxidante y toxicidad del extracto. 


\section{DISCUSIÓN}

A pesar de la percepción generalizada de que los productos vegetales naturales no producen efectos secundarios [47], lo cierto es que muchos productos cosméticos o farmacológicos pueden producir síntomas de carácter leve, como irritación o inflamación del tejido que ha entrado en contacto con el compuesto [27], o problemas de carácter más severo, como retrasos en el desarrollo embrionario [48], carcinogénesis [49], teratogénesis [50], etcétera.

Estos efectos de carácter tóxico pueden tener lugar debido a metabolitos del propio vegetal $[35,37,43]$ o a especies químicas de origen antrópico que el organismo fotosintético absorbe durante su vida $[48,51,52]$.

En primer lugar, al tratarse de extractos brutos en los que no ha habido fraccionamiento, purificación o aislamiento de las diferentes fases, como en otros trabajos con diversas matrices $[19,33,35,53,54]$, los NOAEL y las $\mathrm{LC}_{50}$ obtenidos para cada extracto podrían verse afectados por la mortalidad causada por el pH o la concentración salina de dicho extracto. Estos efectos podrían cuantificarse realizando ensayos toxicológicos con las diferentes fracciones del extracto para detectar aquellas que muestren actividad tóxica [55].

$\mathrm{El} \mathrm{pH}$ puede ser la causa de que las $\mathrm{LC}_{50}$ obtenidas en Artemia-24h sean menores que las observadas en MTT-24h, ya que en este último tipo de ensayo el medio donde se encuentran las células durante el periodo de exposición el medio se encuentra tamponado gracias a una inyección constante de $\mathrm{CO}_{2}$ en la incubadora, a diferencia del ensayo con nauplios de Artemia salina, donde la capacidad de tamponamiento de la solución salina es menor. No obstante, para averiguar si realmente las $\mathrm{LC}_{50}$ de cada extracto Artemia-24h son menores que en el ensayo MTT-24h, sería necesario realizar un screening más afinado entre las concentraciones 1 y $15 \%(v / v)$ del extracto inicial. Además, de esta manera se podrían recalcular los NOAEL y las $\mathrm{LC}_{50}$ de una manera más precisa.

Pese a ello, la $\mathrm{LC}_{50}$ calculada en el ensayo Artemia-24 con dicromato potásico para nauplios en estado instar II fue de 7,7304 $\mu \mathrm{g} / \mathrm{mL}$, similar a la obtenida por otros autores: $7,4 \mu \mathrm{g} / \mathrm{mL}$ [56] y $12,5 \mu \mathrm{g} /$ $\mathrm{mL}$ [46], lo que confirma la correcta sensibilidad de los nauplios utilizados en los ensayos con los extractos y la adecuada preparación de la solución salina utilizada como medio en el ensayo.

Por otro lado, las sales presentes en la matriz pueden ser arrastradas al extracto, haciendo que la concentración de iones de este aumente. Schiener et al. (52) observaron que Palmaria palmata, tras sufrir procesos de biorrefinación con extractantes organoacuosos, puede presentar hasta un $35 \%$ de los cloruros del alga sin refinar. También se vio, en el mismo estudio, que se conservan en porcentajes no menores a un $40 \%$ las sales de arsénico y de yodo, y metales como el zinc, el cobre y el hierro respecto a las cantidades observadas en el alga en bruto, lo que podría aumentar, de manera exógena, la toxicidad de los extractos de P. palmata.

Maruthanayagam et al. [35] observaron que un extracto acuoso de Geitlerinema sp., una cianobacteria, presentaba toxicidad en Artemia salina y en líneas celulares cancerosas. Sin embargo, al fraccionar dicho extracto se descubrió que la toxicidad del extracto solamente se debía a 7 compuestos orgánicos.

Lee, Nishizawa, Shimizu, y Saeki [57] extrajeron la fracción hidrosoluble de P. palmata y a continuación aislaron las ficobilinas del alga. Dichas ficobilinas, que por otro lado presentaban actividad 
antiinflamatoria, no presentaron toxicidad en macrófagos de ratón (RAW264.7) en el rango de 100 a $1000 \mu \mathrm{g} / \mathrm{mL}$.

En este sentido, se puede sospechar que, en parte, la actividad tóxica de los extractos brutos de algas se deba a compuestos e iones que proceden del ambiente en el que vive el alga, y que podrían ser retirados o aislados del extracto con un fraccionamiento adecuado, como en el método utilizado por Harnedy et al. (54) para el aislamiento de péptidos con actividad antioxidante en P. palmata, o la purificación de polisacáridos presentes en Solieria chordalis y P. palmata realizada por Suwal et al. [58].

No obstante, tanto las especies químicas exógenas como el pH o la concentración salina de los extractos de vegetales con capacidad antioxidante son factores que hay que tener en cuenta toxicológicamente si se pretende utilizar el extracto bruto en los ensayos preclínicos, ya que se trata de mezclas complejas de las que no existe suficiente información y no se controlan todos los parámetros fisicoquímicos [14] que pueden favorecer la aparición de los síntomas anteriormente mencionados, que son especialmente peligrosos en el caso de trasplante de órganos [2,59-61].

Dentro de las mezclas complejas que son los extractos brutos vegetales, se ha visto que un screening de los principales grupos químicos potencialmente tóxicos no basta para establecer relaciones entre concentración de estos compuestos y la actividad tóxica que muestra el extracto, pudiendo deberse dicha actividad a especies químicas no detectadas [37], e incluso pudiendo variar entre especies la sensibilidad frente al extracto dependiendo de los compuestos detectados [41]. En el presente estudio, la sensibilidad de los nauplios en estado instar II utilizados en el ensayo Artemia-24h y de las single cells del ensayo MTT-24h frente a los extractos podría diferir, por lo que solo son válidas las comparaciones entre grupos del mismo tipo de ensayo.

También se ha reportado, en otras ocasiones, que la toxicidad de un producto utilizado en cosmética, alimentación o farmacología se deba a residuos del proceso de extracción (62) o al excipiente que acompaña a dicho producto al ser administrado [63-65]. No es el caso de los extractos analizados en el presente estudio, ya que en ninguno de los ensayos el grupo Excipiente, que contenía el posible residuo del extractante puro tras ser rotavaporado y resuspendido, difería significativamente del grupo Control. Por otra parte, no se puede descartar que existan efectos sinérgicos entre el extractante y otros compuestos del alga que aumenten la toxicidad de los segundos, aunque es un factor inherente al método de extracción que igualmente debe ser detectado con los ensayos llevados a cabo.

En lo que respecta a la toxicidad de extractos de las diferentes algas, Álvarez-Gómez, Korbee, Casas-Arrojo, Abdala-Díaz y Figueroa [66] llevaron a cabo un screening toxicológico de extractos organoacuosos en bruto de Hydropuntia cornea y Gracilariopsis longissima, dos algas rojas. Para ello utilizaron como líneas celulares macrófagos de ratón (RAW264.7), fibroblastos gingivales (HGF) y queratinocitos humanos inmortalizados (HaCaT), dando como resultado una $\mathrm{LC}_{50}$ de 259,5 $\mathrm{mg} / \mathrm{mL}$ del extracto liofilizado y resuspendido de $H$. cornea en el caso del ensayo MTT-24h con $\mathrm{HaCaT}$, no obteniéndose datos para este ensayo con el extracto de G. longissima. Con HGF, las $\mathrm{LC}_{50}$ de los extractos de $H$. cornea y $G$. longissima dieron como resultado 250,7 y $4,2 \mathrm{mg} / \mathrm{mL}$ respectivamente, mientras que en RAW264.7 las $\mathrm{LC}_{50}$ observadas fueron 0,12 y $0,41 \mathrm{mg} / \mathrm{mL}$ respectivamente, poniendo de manifiesto la posibilidad de que dos especies de algas rojas puedan mostrar una toxicidad diferente en los diversos tipos celulares con los que puede entrar en contacto, lo que no sucede, en principio, en el estudio realizado con Palmaria palmata y Porphyra sp. De hecho, a pesar de que en el presente estudio los NOAEL y las $\mathrm{LC}_{50}$ están referidos a una concentración $(\%, v / v)$ de extracto 
en porcentaje, tanto en el ensayo MTT-24h como en el ensayo Artemia-24h, estos parámetros indican que el extracto de $P$. palmata es el más tóxico de los dos, manteniéndose las diferencias entre ambos extractos en todo el estudio.

\section{CONCLUSIONES}

A partir de los resultados obtenidos en el presente estudio, podemos concluir que tanto el extracto de Palmaria palmata como el de Porphyra sp. presentan toxicidad en queratinocitos humanos inmortalizados (HaCaT) y en nauplios de Artemia salina.

El extracto de $P$. palmata es el más tóxico, presentando un NOAEL de $<1 \%$ y una $\mathrm{LC}_{50}$ de $7,8743 \%$ del extracto inicial en el ensayo MTT-24h con queratinocitos humanos inmortalizados (HaCaT), y un NOAEL de $<1 \%$ y una $\mathrm{LC}_{50}$ de 3,2583 \% del extracto inicial en el ensayo Artemia-24h.

Por otro lado, el extracto de Porphyra sp. es el menos tóxico, presentando un NOAEL de $1 \%$ y una $\mathrm{LC}_{50}$ de 10,7463\% del extracto inicial en el ensayo MTT-24h con queratinocitos humanos inmortalizados (HaCaT), y un NOAEL de $5 \%$ y una $\mathrm{LC}_{50}$ de 6,5688 \% del extracto inicial en el ensayo Artemia-24h.

Los Índices de Eficiencia Antioxidante (IEA) para cada ensayo y cada parámetro toxicológico mostraron que el extracto de Porphyra sp. muestra una mayor actividad antioxidante a la vez que presenta una menor toxicidad que en el caso del extracto de Palmaria palmata. Por tanto, sería deseable escoger el extracto de Porphyra sp. como candidato para continuar los ensayos preclínicos en el desarrollo de un fármaco eficaz para el tratamiento de la lesión por isquemia/reperfusión.

\section{AGRADECIMIENTOS}

A la Universidad Católica de Valencia San Vicente Mártir (UCV) y al Instituto de Medio Ambiente y Ciencia Marina (IMEDMAR).

\section{REFERENCIAS BIBLIOGRÁFICAS}

[1] Arumugam TV, Shiels IA, Woodruff TM, Granger DN, Taylor SM. The role of the complement system in ischemia-reperfusion injury. Shock. 2004;21(5):401-9.

[2] Ponticelli C. Ischaemia-reperfusion injury: A major protagonist in kidney transplantation. Nephrol Dial Transplant. 2014;29(6):1134-40.

[3] Salvadori M, Rosso G, Bertoni E. Update on ischemia-reperfusion injury in kidney transplantation: Pathogenesis and treatment. World J Transplant. 2017;5(2):52.

[4] Kaskel FJ, Feuerstein D, Castaneda MP, Swiatecka-Urban A, Devarajan P, Tellis V, et al. Activation of Mitochondrial Apoptotic Pathways in Human Renal Allografts After Ischemia-Reperfusion Injury. Transplantation. 2003;76(1):50-4. 
[5] Saat TC, van den Akker EK, IJzermans JNM, Dor FJMF, de Bruin RWF. Improving the outcome of kidney transplantation by ameliorating renal ischemia reperfusion injury: Lost in translation? J Transl Med. 2016;14(1):1-9.

[6] Belda-Antolí M, Padrón-Sanz C, Cejalvo-Lapeña D, Prieto-Moure B, Lloris-Cejalvo JM, Lloris-Carsí JM. Antioxidant potential of Himanthalia elongata for protection against ischemia-reperfusion injury in the small bowel. Surg (United States). 2017;162(3):577-85.

[7] Guerra JIE. Elejalde Guerra JI. Estrés oxidativo, enfermedades y tratamientos antioxidantes. An Med Interna. 2001;18:326-35.

[8] Eltzschig HK, Eckle T. Ischemia and reperfusion-from mechanism to translation. Nat Med. 2011;17(11):1391-401.

[9] Gupta DK, Palma JM, Corpas FJ, editors. Antioxidants and Antioxidant Enzymes in Higher Plants. Primera ed. Cham, Suiza: Springer Nature; 2018.

[10] Valko M, Leibfritz D, Moncol J, Cronin MTD, Mazur M, Telser J. Free radicals and antioxidants in normal physiological functions and human disease. Int J Biochem Cell Biol. 2007;39(1):4484.

[11] Pryor WA, Cornicelli JA, Devall LJ, Tait B, Trivedi BK, Witiak DT, et al. A Rapid Screening Test To Determine the Antioxidant Potencies of Natural and Synthetic Antioxidants. J Org Chem. 1993;58(13):3521-32.

[12] Farag RS, El-Baroty GS, Basuny AM. The influence of phenolic extracts obtained from the olive plant (cvs. Picual and Kronakii), on the stability of sunflower oil. Int J Food Sci Technol. 2003;38(1):81-7.

[13] McCarthy TL, Kerry JP, Kerry JF, Lynch PB, Buckley DJ. Evaluation of the antioxidant potential of natural food/plant extracts as compared with synthetic antioxidants and vitamin e in raw and cooked pork patties. Meat Sci. 2001;58(1):45-52.

[14] Pokorný J. Are natural antioxidants better -and safer- than synthetic antioxidants? Eur J Lipid Sci Technol. 2007;109(6):629-42.

[15] Wang Y, Li W, Xu S, Hu R, Zeng Q, Liu Q, et al. Protective skin aging effects of cherry blossom extract (Prunus yedoensis) on oxidative stress and apoptosis in UVB-irradiated HaCaT cells. Cytotechnology. 2019;7.

[16] Barreira JCM, Morais AL, Ferreira ICFR, Oliveira MBPP. Insights on the formulation of herbal beverages with medicinal claims according with their antioxidant properties. Molecules. 2013;18(3):2851-63.

[17] He C, Xing J, Jiang W, Wang W, Zeng C, Yang X. Pretreatment with Total Flavonoid Extract from Dracocephalum moldavica L. Attenuates Ischemia Reperfusion-induced Apoptosis. Sci Rep. 2018;8(1):1-14.

[18] Petkovic A, Jeremic J, Jakovljevic V, Sobot T, Zivkovic V, Bradic J, et al. Protective Effects of Galium verum L. Extract against Cardiac Ischemia/Reperfusion Injury in Spontaneously Hypertensive Rats. Oxid Med Cell Longev. 2019;2019:1-11.

[19] Takayama C, Luiz-Ferreira A, Vilegas W, Salvador MJ, Almeida ACA, Dunder RJ, et al. Antioxidant Action of Mangrove Polyphenols against Gastric Damage Induced by Absolute Ethanol and Ischemia-Reperfusion in the Rat. Sci World J. 2012;2012:1-9. 
[20] Viswanatha GL, Venkataranganna MV. Methanolic leaf extract of Punica granatum attenuates ischemia-reperfusion brain injury in Wistar rats: Potential antioxidant and anti-inflammatory mechanisms. 2018;(13).

[21] Zeng K-W, Tu P-F, Liu L-Y, Jiang Y, Song W-T, Liu Y-Q, et al. The Ethanolic Extract of Caesalpinia sappan Heartwood Inhibits Cerebral Ischemia/Reperfusion Injury in a Rat Model Through a Multi-Targeted Pharmacological Mechanism. Front Pharmacol. 2019;10(February):1-15.

[22] Li J, Zhang QH, Li S, Dai W, Feng J, Wu L, et al. The natural product fucoidan ameliorates hepatic ischemia-reperfusion injury in mice. Biomed Pharmacother. 2017;94:687-96.

[23] Shobha JC, Kutala VK, Naidu MU, Parinandi NL, Tridandapani S, Kuppusamy P, et al. C-phycocyanin protects against ischemia-reperfusion injury of heart through involvement of p38 MAPK and ERK signaling. Am J Physiol Circ Physiol. 2005;290(5):H2136-45.

[24] Zhu H, Tian J, Li C, Gao Y, Xing Y, Shen J. Fucoidan, a sulfated polysaccharide from brown algae, against myocardial ischemia-reperfusion injury in rats via regulating the inflammation response. Food Chem Toxicol. 2011;49(9):2090-5.

[25] Qin SG, Tian HY, Wei J, Han ZH, Zhang MJ, Hao GH, et al. 3-bromo-4,5-dihydroxybenzaldehyde protects against myocardial ischemia and reperfusion injury through the akt-PGC1 $\alpha$-Sirt3 pathway. Front Pharmacol. 2018;9(JUL):1-9.

[26] Sun C, Wu F, Chen D, Ge J. Therapeutic effects of polysaccharides extracted from Porphyra yezoensis in rats with cerebral ischemia/reperfusion injury. Arch Biol Sci. 2018;70(2):233-9.

[27] Campos PMBGM, Benevenuto CG, Calixto LS, O. Melo M, Pereira KC, Gaspar LR. Spirulina, Palmaria palmata, Cichorium intybus, and Medicago sativa extracts in cosmetic formulations: an integrated approach of in vitro toxicity and in vivo acceptability studies. Cutan Ocul Toxicol. 2019;9527:1-25.

[28] Hartung T. From alternative methods to a new toxicology. Eur J Pharm Biopharm. 2011;77(3):33849.

[29] Xu JJ, Urban L. The human predictive value of combined animal toxicity testing. In: Xu JJ, Urban L, editors. Predictive Toxicology in Drug Safety. Primera ed. New York, EE. UU.: Cambridge University Press. 2011;1-17.

[30] Wilson VG. Growth and Differentiation of HaCaT Keratinocytes. Methods Mol Biol. 2016;1341:257-84.

[31] Li L, Huang T, Lan C, Ding H, Yan C, Dou Y. Protective effect of polysaccharide from Sophora japonica L. flower buds against UVB radiation in a human keratinocyte cell line (HaCaT cells). J Photochem Photobiol B Biol. 2019;191:135-42.

[32] Turkoglu M, Pekmezci E, Kilic S, Dundar C, Sevinc H. Effect of Ficus carica leaf extract on the gene expression of selected factors in HaCaT cells. J Cosmet Dermatol. 2017;16(4):e54-8.

[33] Ji D, You L, Ren Y, Wen L, Zheng G, Li C. Protective effect of polysaccharides from Sargassum fusiforme against UVB-induced oxidative stress in HaCaT human keratinocytes. J Funct Foods. 2017;36:332-40.

[34] Kim S, You DH, Han T, Choi EM. Modulation of viability and apoptosis of UVB-exposed human keratinocyte HaCaT cells by aqueous methanol extract of laver (Porphyra yezoensis). J Photochem Photobiol B Biol. 2015;141:301-7. 
[35] Maruthanayagam V, Nagarajan M, Sundararaman M. Cytotoxicity assessment of cultivable marine cyanobacterial extracts in Artemia salina (brine shrimp) larvae and cancer cell lines. Toxin Rev. 2013;32(1):1-9.

[36] Zubia M, Fabre MS, Kerjean V, Deslandes E. Antioxidant and cytotoxic activities of some red algae (Rhodophyta) from Brittany coasts (France). Bot Mar. 2009;52(3):268-77.

[37] Sanabria-Galindo A, López SI, Gualdrón R. Estudio fitoquimico preliminar y letalidad sobre Artemia salina de plantas colombianas. Rev Colomb Cienc Quím Farm. 1997;26(1):15-9.

[38] Lopes G, Silva TMS da, Echevarria A. Toxicity from Crude Extracts and Glycoalkaloid Fractions of Solanum spp. Against Artemia salina and Biomphalaria glabrata. Rev Virtual Química. 2016;8(1).

[39] Charoenphon N, Kangwanrangsan N, Jiraungkoorskul W. Artemia salina lethality and histopathological studies on bacopa monnieri leaf extract. Indian J Anim Res. 2018;52(4):610-4.

[40] Rocha-Filho CAA, Albuquerque LP, Silva LRS, Silva PCB, Coelho LCBB, Navarro DMAF, et al. Assessment of toxicity of Moringa oleifera flower extract to Biomphalaria glabrata, Schistosoma mansoni and Artemia salina. Chemosphere. 2015;132:188-92.

[41] Kim YD, Choi JS. Larvicidal effects of korean seaweed extracts on brine shrimp Artemia salina. J Anim Plant Sci. 2017;27(3):1039-46.

[42] Blois M. Antioxidant Determinations by the Use of a Stable Free Radical. Nature. 1958;181(4617):1199-200.

[43] Kiviranta J, Abdel-Hamed A. Toxicity of the blue-green alga Oscillatoria agardhii to the mosquito Aedes aegypti and the shrimp Artemia salina. World J Microbiol Biotechnol. 1994;10:51720.

[44] Kiviranta J, Sivonen K, Niemelä SI, Huovinen K. Detection of toxicity of cyanobacteria by $A r$ temia salina bioassay. Environ Toxicol Water Qual. 1991;6(4):423-36.

[45] Sarabia R. Toxicidad y acumulación de cadmio en poblaciones de diferentes especies de Artemia. Tesis Doctoral. Facultad de ciencias biológicas. Universidad de Valencia; 2002.

[46] González Y, Gilling PA. Determinación de la toxicidad aguda del dicromato de potasio en larvas de Artemia salina. Anu Toxicol. 2001;1(1):104-8.

[47] Wagemaker TAL, Rijo P, Rodrigues LM, Maia Campos PMBG, Fernandes AS, Rosado C. Integrated approach in the assessment of skin compatibility of cosmetic formulations with green coffee oil. Int J Cosmet Sci. 2015;37(5):506-10.

[48] Bai SH, Ogbourne SM. Glyphosate: environmental contamination, toxicity and potential risks to human health via food contamination. Environ Sci Pollut Res. 2016;23(19):18988-9001.

[49] Man S, Wang G, Liu C, Gao W. Annual advance of traditional medicine toxicity in 2018. TMR. 2018;4(3):111-7.

[50] Nobakht M, Fattahi M, Hoormand M, Milanian I, Rahbar N, Mahmoudian M. A study on the teratogenic and cytotoxic effects of safflower extract. J Ethnopharmacol. 2000;73(3):453-9.

[51] Parks AN, Portis LM, Schierz PA, Washburn KM, Perron MM, Burgess RM, et al. Bioaccumulation and toxicity of single-walled carbon nanotubes to benthic organisms at the base of the marine food chain. Environ Toxicol Chem. 2013;32(6):1270-7. 
[52] Schiener P, Zhao S, Theodoridou K, Carey M, Mooney-McAuley K, Greenwell C. The nutritional aspects of biorefined Saccharina latissima, Ascophyllum nodosum and Palmaria palmata. Biomass Convers Biorefinery. 2017;7(2):221-35.

[53] Bengtström L, Trier X, Granby K, Rosenmai AK, Petersen JH. Fractionation of extracts from paper and board food contact materials for in vitro screening of toxicity. Food Addit Contam Part A Chem Anal Control Expo Risk Assess. 2014;31(7):1291-300.

[54] Harnedy PA, O'Keeffe MB, FitzGerald RJ. Fractionation and identification of antioxidant peptides from an enzymatically hydrolysed Palmaria palmata protein isolate. Food Res Int. 2017;100:416-22.

[55] Cedergreen N. Quantifying synergy: A systematic review of mixture toxicity studies within environmental toxicology. PLoS One. 2014;9(5).

[56] Sorgeloos P, Remiche-Van Der Wielen C, Persoone G. The use of Artemia nauplii for toxicity tests - A critical analysis. Ecotoxicol Environ Saf. 1978;2(3-4):249-55.

[57] Lee D, Nishizawa M, Shimizu Y, Saeki H. Anti-inflammatory effects of dulse (Palmaria palmata) resulting from the simultaneous water-extraction of phycobiliproteins and chlorophyll a. Food Res Int. 2017;100(June):514-21.

[58] Suwal S, Perreault V, Marciniak A, Tamigneaux É, Deslandes É, Bazinet L, et al. Effects of high hydrostatic pressure and polysaccharidases on the extraction of antioxidant compounds from red macroalgae, Palmaria palmata and Solieria chordalis. J Food Eng. 2019;252:53-9.

[59] Nashan B, Abbud-Filho M, Citterio F. Prediction, prevention, and management of delayed graft function: where are we now? Vol. 30, Clinical Transplantation. 2016:1198-1208.

[60] Smith SF, Hosgood SA, Nicholson ML. Ischemia-reperfusion injury in renal transplantation: 3 key signaling pathways in tubular epithelial cells. Kidney Int. 2019;95(1):50-6.

[61] Wu WK, Famure O, Li Y, Kim SJ. Delayed graft function and the risk of acute rejection in the modern era of kidney transplantation. Kidney Int. 2015;88(4):851-8.

[62] Tran BN, Richard O, Robin S, Robert J, Aldous KM. Use of methanol for the efficient extraction and analysis of melamine and cyanuric acid residues in dairy products and pet foods. J Agric Food Chem. 2010;58(1):101-7.

[63] Masi S, De Cléty SC, Anslot C, Detaille T. Acute amiodarone toxicity due to an administration error: Could excipient be responsible?: Letter to the Editors. Br J Clin Pharmacol. 2009;67(6):691-3.

[64] Osterberg RE, See NA. Toxicity of Excipients - A Food and Drug Administration Perspective. Int J Toxicol. 2003;22(5):377-80.

[65] Scherließ R. The MTT assay as tool to evaluate and compare excipient toxicity in vitro on respiratory epithelial cells. Int J Pharm. 2011;411(1-2):98-105.

[66] Álvarez-Gómez F, Korbee N, Casas-Arrojo V, Abdala-Díaz RT, Figueroa FL. UV photoprotection, cytotoxicity and immunology capacity of red algae extracts. Molecules. 2019;1-16. 
